\title{
Performance of Channel Estimation Schemes in the presence of Gaussian Mixture Model
}

\author{
Saveeta Bai ${ }^{1}$, Abid Muhammad Khan ${ }^{2}$, Muhammad Rauf ${ }^{3}$, Suresh Kumar ${ }^{4}$ and Haresh Kumar $^{5}$ \\ 1.2.5 Electronic Engineering Department, Sir Syed University of Engineering and Technology, \\ Karachi, Pakistan ${ }^{3}$ Department of Electronic Engineering, Dawood University of Engineering \\ and Technology, Pakistan ${ }^{4}$ Department of Electronic Engineering, Universitat Politècnica de \\ Catalunya, Barcelona, Spain \\ saveetahari4@gmail.com, abidmk@ssuet.edu.pk,muhammad.rauf@duet.edu.pk, \\ suresh.kkes@gmail.com, hkumar@ssuet.edu.pk \\ Corresponding author: Muhammad.rauf@duet.edu.pk
}

\begin{abstract}
Channel estimation (CE) plays a crucial role in establishing a wireless link, specifically at the receiver node. Most of the receivers that estimate the channel is in the presence of AWGN. However, these schemes perform expressively worse when the impulsive noise is added in AWGN which is introduced by manmade sources (pressure cooker, motorbike, electric supply) as well as natural noises (earthquakes and thundering). The major contribution of this research is to analyze the channel estimation schemes in the Gaussian mixture model (GMM) environment. The performance of channel estimation schemes has been compared in terms of mean square error (MSE) and bit error rate (BER). Four channel estimation schemes e.g., MMSE, DFT, correlation- based methods like Gauss-Seidel (GS) and Successive OverRelaxation (SOR), are studied and analyzed. The study reveals that the correlation scheme based on the method of SOR is more effective as compared to the methods of DFT, MMSE and GS because of faster convergence rate along with the minimum number of iteration. SOR shows sustainable results up to the probability of an impulsive element of 5 Percent.
\end{abstract}

Keywords: GMM, LS, MMSE, DFT, GS, SOR.

\section{Introduction}

In the wireless communication system, due to the multipath effects, several complexities are associated with the transmitted signals such as reflection, scattering, and diffraction [1]. In most of the research, AWGN channel models are utilized for many wireless applications but whenever impulse noise occurs in the AWGN channel model, it adversely affects the wireless system. Thus, the synchronization and the estimation of the channel in the GMM (Gaussian mixture model) environment are among the highly complicated, yet vital issues in the advanced wireless communication system.

The four different approaches that are used in channel estimation which are as follows. First is the preamble-based approach which is stated as the simplest way is to sound the channel with known wideband noise like signal and listen to it at all frequencies. Equivalently, one can transmit an impulse signal and obtain its impulse response. This is how a preamble-based approach is performed. Preamble based approach is useful where channel variation is slow, in other words, slow fading channel [2]. The second approach is the pilot-based. In this approach, pilots are transmitted together with data, and channel variation can be tracked by the symbol [3, 4]. Hence, it is useful for the fast fading channel as well. In the pilot-based approach, one can transmit known pilots at selective frequencies and obtain responses at those frequencies. Then these responses are interpolated by different methods. Yet the third approach can be called the totally blind approach, where known measurements of the signals are used for channel estimation. The totally blind approach is suitable for applications where bandwidth is minimal and saves the training overhead $[5,6]$. On the contrary side, it has a negative factor of being exceedingly computational. Semi blind approaches fill in between, is known as the fourth one.

Received: May 29 ${ }^{\text {th }}, 2021$. Accepted: September $28^{\text {th }}, 2021$

DOI: 10.15676/ijeei.2021.13.3.10 
Frequency domain (FD) and Time domain (TD) are the main two domains are used for the characterization of Channel estimation. In the channel estimation scheme based on frequency domain (FD), channel frequency response (CFR) is assessed at each pilot. Subsequently, interpolation takes place by different techniques. Least Squares (LS) and Minimum Mean Squares Errors (MMSE) are utilized by the popular frequency domain (FD). In the Least Squares based, CFR can be simply stated as the ratio between output and input signal at pilot frequencies. Therefore, it is known as the facile estimation technique while inviting the issue of distortion. This flaw is addressed by MMSE, at the cost of complexity enhancement. In MMSE, MSE (average of the squares of the difference between the estimator and what is estimated) is minimized by the prior knowledge of distortion variance.

However, the TD channel estimation schemes are divided into discrete Fourier transforms (DFT) and correlation schemes. In the TD channel estimation strategy, the channel impulse response (CIR) is estimated first. Then, the estimated response is moved through a fast Fourier transform (FFT) operation for equalization of the channel at each subcarrier in the FD.

In the TD scheme, the correlation method is further classified into three types. Correction Error Cancellation (CEC), GS method and, Successive SOR. In [7], FD pilot and TD processing (FPTP) method is presented, in this method correlation error cancellations (CEC) technique is applied. In spite of being popular and efficient, the performance of mean square error is adversely affected when any of its neighboring routes are near to the strength of the strongest route. Consequently, additional computation power is required by FPTP, in the form of iteration in CEC. In [8], the author utilizes the GS method is suitable for less number of guard bands. When the number of guard bands are increased, the number of iterations will also increase as the consequences the complexity also goes up. In addition, if channel conditions are unknown then the number of iterations are also increased in Gauss-Seidel method. However, the SOR based model is more efficient as compared to the GS based model with less number of iterations. Thus, this paper presents SOR method. In the SOR, a few numbers of iterations are required to converge to a precise solution with unknown initial values [9-12].

Moreover, the literature states that several studies have paid attention to the assumption of AWGN noise models $[13,14]$ commonly these suppositions are beneficial for some applications nevertheless some distortions in the background exist practically which are improperly modeled due to the AWGN noise. At the point when the noise goes diverge from Gaussian, it affects the existing channel estimation techniques abruptly because the Gaussian-based estimation techniques are susceptible to noise. In this context the renowned noise model such as GMM presents impulsive noise known as non-Gaussian noise. The comprehensive domain of nonGaussian noise distribution, is also identified by this model along this it also incorporates most of the noise types which is available in a variety of wireless communication systems [15-22].

In the OFDM system, the frequency-domain (FD) channel estimation schemes have gained much more popularity than time-domain schemes. In the frequency-domain approaches pilots are inserted in the FD and it easy to estimate channel using interpolation. However, the presented time-domain correlation-based channel estimation technique differs from frequency-domain estimators in such way that it does not depend on interpolation [23, 24]. In the correlation method by increasing or decreasing the number of pilot or pilot spacing, results would not be affected while in the interpolation method errors would occur due to the variation of the number of pilots and pilot spacing.

In this paper DFT, MMSE GS, and SOR methods are presented in the GMM environment where the SOR method is adopted expressively rather than the GS method. Because in the GS method when the number of guard bands increase the convergence rate or the number of iteration also increases. In addition, the MSE and BER are improved in the SOR method as compared to the GS method. This article is structured in the following sections. The overview of the GMM based OFDM system model is explained in Section 2. In Section 3 the conventional channel and proposed channel estimation techniques are discussed. Section 4 defines the simulation parameters that are used in this work. Section 5 includes the Results and analysis. Lastly, the 
conclusion of this research work is presented in Section 6 whereas last section contains the references.

\section{System Model}

The system block diagram of the frequency and time-domain channel estimation model is shown in Figure 1 [25]. The model begins with the generation of pilots, data subcarriers $P[K]$ and $D[K]$ respectively. Subsequently in mux block theses subcarriers are added and give the frequency-domain samples $X[K]$. Then Inverse Fast Fourier is applied on $X[K]$ samples that transforms FD samples $X[K]$ into TD $x(n)$, which is given as:

$$
x(n)=\operatorname{IFFT}\{X[K]\}=\sum_{k=0}^{N-1} X[K] e^{\frac{j 2 \pi k n}{N_{F F T}}}
$$

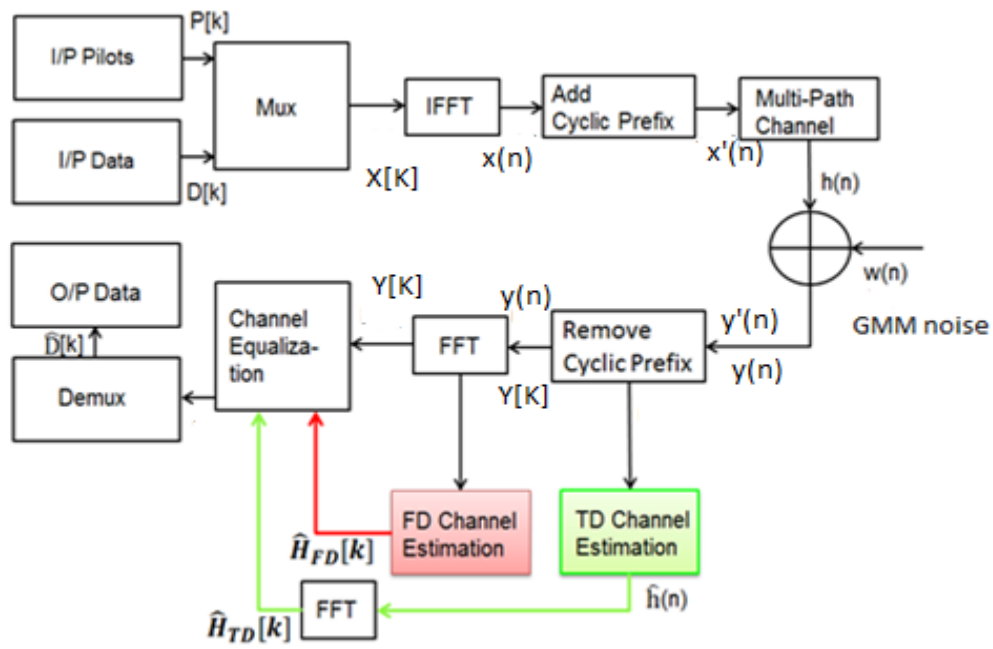

Figure 1. System block diagram of FD CE and TD CE

Where $N_{F F T}$ signifies the number of Fast Fourier Transform. To eliminate inter-symbol interference, cyclic prefix $N_{g}$ are inserted in each OFDM symbol and samples become $x^{\prime}(n)$, that can be stated as:

$$
x^{\prime}(n)=\left\{\begin{array}{c}
x\left(N_{F F T}+n\right), \quad n=-N_{G}+1 \ldots,-1 \\
x(n), \quad n=0,1, \ldots \ldots, N_{F F T}-1
\end{array}\right.
$$

When the received signal travels through the frequency selective multipath channel, it becomes

$$
\begin{aligned}
& x^{\prime}(n) \\
& y(n)=x^{\prime}(n) \otimes h(n)+w(n)
\end{aligned}
$$

Here the additive Gaussian mixture noise is signifies by the term $w(n)$. The impulse response of multi-path channel $h(n)$ can be stated as:

$$
h(n, \tau)=\sum_{i=0}^{L-1} h_{i} e^{j\left(\frac{2 \pi}{N_{F F T}}\right) f_{D_{i}} T n} \delta\left(\tau-\tau_{i}\right)
$$

where $h_{i}$ represents the $i^{\text {th }}$ complex part of path gain, $f_{D_{i}}$ is the $i^{t h}$ path Doppler frequency shift, $\tau_{i}$ is the route delay of matching normalized and the term $L$ shows the entire number of channel taps. Without losing the simplification, there is a possibility to use a model having low-pass system and therefore the received signal is $y^{\prime}(n)$ with cyclic prefix that would be $y(n)$ after removal of the cyclic prefix, can be represented as:

$$
y(n)=\sum_{m=0}^{N_{F F T}-1} h(m)\left[d((n-m))_{N_{F F T}}+p((n-m))_{N_{F F T}}\right]+w(n)
$$

where $m$ is the indexing used in TD, $(.)_{N_{F F T}}$ represents the modulo of $N_{F F T}$ and, $w(n)$ is GMM noise. When FFT is applied to Equation (5) it would be rewritten as: 


$$
\begin{aligned}
& Y[k]=F F T\{y(n)\}=\frac{1}{N_{F F T}} \sum_{n=0}^{N_{F F T}-1} y(n) e^{\frac{j 2 \pi k n}{N_{F F T}}} \\
& n=1,2, \ldots . N_{F F T}-1
\end{aligned}
$$

When considering the much smaller length of CIR than the guard band interval as consequences there is no ISI.

The response of $Y[k]$ can be represented as:

$$
Y[k]=X[k] H[k]+W[k]
$$

$W[k]$ and $H[k]$ are the Fourier transform of $w(n)$ and $h(n)$ respectively. Where $w(n)$ can be defined by GMM [26], distribution as:

$$
p(w(n))=(1-\emptyset) \cdot \mathcal{C N}\left(0, \sigma_{n}^{2}\right)+\emptyset \cdot \mathcal{C N}\left(0, T \sigma_{n}^{2}\right)
$$

where $T \gg 1$ represent magnitude of the impulsive-noise and $\mathcal{C N}\left(0, T \sigma_{n}^{2}\right)$ represents the distribution of Gaussian signal having zero mean and variance $\sigma_{n}^{2}$, and to get control over the noise level in GMM model $\emptyset$ is the mixture parameter. As per Equation (8), it can be inferred that stronger GMM noise by higher noise variance $T \sigma_{n}^{2}$ as well as higher mixture parameter $\emptyset$. Thus, the variance of GMM is obtained as:

$$
\sigma_{z}^{2}=(1-\emptyset) \sigma_{n}^{2}+\emptyset T \sigma_{n}^{2}
$$

\section{Conventional Channel Estimation Techniques}

Three different types of conventional CE techniques are presented in this paper. These are as follow DFT, MMSE, and Correlation methods. Consider a system model in FD systems by using Equation (7), such as:

$$
Y[k]=X[k] H[k]+W[k] \quad 0 \leq k \leq N_{F F T}-1
$$

where $X$ and $Y$ represent transmitted and received FD signals at each subcarrier respectively. However, $H$ and $W$ represent the channel transfer function and GMM noise for OFDM symbol, respectively.

\section{A. MMSE Evaluation}

In MMSE, CE requires the prior familiarity of auto covariance matrix $R_{H H}$ and noise variance $\sigma_{N}^{2}$. Let the error in channel estimation $e$ as:

$e=H-\widehat{H}$

Now, MSE can be defined as the average of the squares of the difference between the estimators and what is estimated:

$$
E\left\{|e|^{2}\right\}=E\left\{|H-\widehat{H}|^{2}\right\}
$$

The MMSE-based channel estimation can be simplified as [27-30]:

$$
\widehat{H}_{M M S E}=R_{H H}\left(R_{H H}+\sigma_{N}^{2}\left(X^{H} X\right)^{-1}\right)^{-1} \widehat{H}_{P, L S}
$$

where auto covariance matrixes of $H$ is denoted by $R_{H H}$. The performance of MMSE estimator is better as compared to LS [31]. However, because of the inversion of the matrix in Equation (13), it has high computational complexity as compared to LS.

\section{B. DFT Evaluation}

In the TD DFT method, the CE is first obtained in the FD as usual, and then it is transformed into time-domain in form of inverse-DFT where noise is removed from all the time locations where channel taps are not present. After cleaning, the estimations are transformed back to the FD using DFT. The DFT-based algorithm provides enhanced estimation precision when compared with orthodox LS and MMSE estimators since it permits decrease of noise outside the most extreme channel delay length $D$. DFT-based algorithm can be done in the additional advances:

- Use Calculate $\widehat{H}_{L S}[k]$, the $L S$-based channel transfer function as:

$$
\widehat{H}_{-} L S[k]=Y[k] / X[k]=H[k]+W[k] / X[k]
$$

- Convert $\widehat{H}_{L S}[k]$ into time-domain and use IDFT. 


$$
\operatorname{IDFT}\left\{\widehat{H}_{L S}[k]\right\} \triangleq \widehat{h}(n)=h(n)+w(n)
$$

Here actual and estimated CIR are represented by $h(n)$ and $\hat{h}(n)$ respectively and $w(n)$ represents the time-domain noise component.

- Minimize the effect of noise in TD by defining coefficients for maximum channel delay length $D$ as:

$$
\hat{h}_{D F T}(n)=\left\{\begin{array}{cc}
h(n)+w(n) & n=0,1 \ldots D-1 \\
0 & \text { otherwise }
\end{array}\right.
$$

- Use Convert $\hat{h}_{D F T}(n)$ into FD by using DFT.

$$
\widehat{H}_{D F T}[k]=\operatorname{DFT}\left\{\hat{h}_{D F T}(n)\right\}
$$

However, DFT suffers leakage of energy in non-significant channel taps when $(n \geq D)$, which can be minimized in the proposed TD CE [25], as shown in Figure 2.

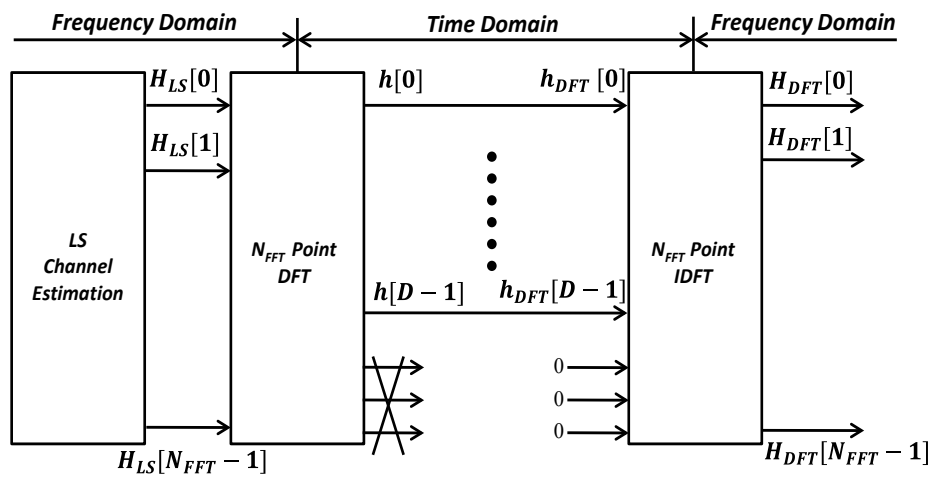

Figure 2. Channel estimation based on DFT

\section{Proposed Correlation Method (SOR)}

In this section, first the brief review of Gauss-Seidel iterative method is explained then its extension i.e. SOR method with the relaxation factor is discussed.

\section{C.1. Gauss-Seidel method}

To estimate channel impulse response, Gauss-Seidel iterative method can be used. However, the convergence rate of Gauss-Seidel is high due to having a large number of iteration. Therefore, to improve the convergence rate of Gauss-Seidel, a proper initial guess such as component without windowing aspect can be utilized. Consequently, the cyclic correlation is taken with the locally generated $p_{1}(n)$ for the received signal $y(n)$,

$$
C_{y p_{1}}(n)=h(n) \otimes\left[C_{d p_{1}}(n)+C_{p_{1} p_{1}}(n)+C_{p_{2} p_{1}}(n)\right]+C_{w p_{1}}(n)
$$

In general $C_{x y}(n)$ is the cyclic correlation between $x$ and $y$. From it is know that $C_{d p_{1}}(n)=0$. Therefore, the response of cyclic correlation becomes,

$$
C_{y p 1}(n)=h(n) \otimes\left[C_{p_{1} p_{1}}(n)+C_{p_{2} p_{1}}(n)\right]+C_{w p_{1}}(n)
$$

It can be shown that both the real and imaginary components of the cross-correlation of noise $w(n)$ with $p_{1}(n), C_{w p_{1}}(l)$, can be estimated as zero mean based Gaussian mixture noise and variance $\sigma_{z}^{2}$. Also, $C_{w p_{1}}(l)=\widetilde{w}(l)$. Therefore, Equation (19) may be formed as:

$$
\begin{aligned}
& C_{y p_{1}}(l)=h(l) \otimes\left[C_{p_{1} p_{1}}(l)+C_{p_{2} p_{1}}(l)\right]+\widetilde{w}(l) \\
& 0 \leq l \leq N_{P}-1
\end{aligned}
$$

Here it is noticed that over the extent of $N_{P}, C_{p_{1} p_{1}}(0)$ and $C_{p_{2} p_{1}}(0)$ behave like delta functions. Thus, In the context of convolution sum the Equation (20) can be restricted as:

$$
C_{y p_{1}}(l)=h(l) \cdot\left[C_{p_{1} p_{1}}(0)+C_{p_{2} p_{1}}(0)\right]+
$$




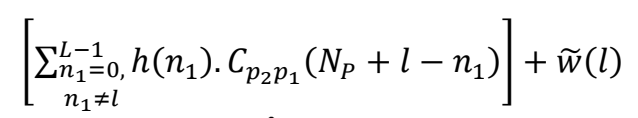

To estimate the CIR $\hat{h}(l)$, one can write Equation (21), as:

$$
\hat{h}(l)=\frac{\begin{array}{c}
C_{y p_{1}}(l)-\sum_{n_{1}=0,}^{L-1} h_{n_{1}} \cdot C_{p_{2} p_{1}}\left(N_{P}+l-n_{1}\right)-\widetilde{w}(l) \\
n_{1} \neq l
\end{array}}{C_{p_{1} p_{1}}(0)+C_{p_{2} p_{1}}(0)}
$$

The estimated initial guess can be written as $\hat{h}_{l}^{(k)}$ :

$$
\hat{h}_{l}^{(0)}=\left[\hat{h}_{0}^{(0)}, \hat{h}_{1}^{(0)}, \ldots . \hat{h}_{n-1}^{(0)}\right]=[0,0, \ldots 0],
$$

Substitute the values of $\hat{h}_{l}^{k}$ in Equation (23) to find the new estimates as $\hat{h}_{l}^{k+1}$.

$$
\hat{h}_{l}^{(K+1)}=\hat{h}_{l}^{k}-\frac{\begin{array}{c}
\sum_{n_{1}=0,}^{L-1} \hat{h}_{1} \\
n_{1} \neq l
\end{array}}{C_{p_{1} p_{1}}(0)+C_{p_{2} p_{1}}\left(N_{p_{2}}-l-n_{1}\right)}
$$

\section{C.2. Successive Over-Relaxation method}

The SOR method starts with the extension of Equation (24) of GS method that contain the relaxation factor $\omega$.

$$
\hat{h}_{l}^{(k+1)}=\hat{h}_{l}^{(k)}+\omega\left(\hat{h}_{l}^{(k+1)}-\hat{h}_{l}^{(k)}\right)
$$

The relaxation factor affects the performance of SOR in a large extent. For instance, if $\omega=1$ the SOR method becomes Gauss-Seidel method. For over-relaxation $1<\omega<2$ for under relaxation $0<\omega<1$. In proposed method, $\omega=1.25$.

After each iteration check if the difference in error is smaller than the threshold value.

$$
\epsilon_{r}=\left|\frac{\widehat{h}_{l}^{(k+1)}-\widehat{h}_{l}^{(k)}}{\widehat{h}_{l}^{(k+1)}}\right|
$$

Until achieving the smaller difference in errors as compared to tolerance $\epsilon_{r}$, the iteration process is continued. In the presented work $\epsilon_{r}=0.001$.

\section{Simulation Parameters}

In this paper, orthodox CE techniques (MMSE and DFT) are compared with the correlation methods (GS and SOR) in the GMM environment for typical urban reception (TU6), the OFDM simulation parameters has been shown in Table 1. In the presented work the cross correlation between data and pilots subcarriers are zero, therefore is no performance degradation is observed

2. Table 1. Simulation Parameters of OFDM

\begin{tabular}{|c|c|}
\hline Parameters & OFDM \\
\hline FFT Size & 1,024 \\
\hline No. of used Data Subcarriers & 960 \\
\hline No. of Pilot Subcarriers & 64 \\
\hline Pilot Spacing & 16 \\
\hline Cyclic Prefix or Guard Time $\left(\mathrm{N}_{\mathrm{G}}\right)$ & $1 / 16$ \\
\hline Signal Constellation $\mathrm{D}[\mathrm{k}]$ & BPSK \\
\hline FFT Sampling Frequency $\left(\mathrm{F}_{\mathrm{S}}\right)$ & $9.142 \times 1 \mathrm{E}^{6}$ \\
\hline Bandwidth $\left(\mathrm{MH}_{\mathrm{z}}\right)$ & 8 \\
\hline Pilot Pattern & 16 \\
\hline Speed of Receiver & $50 \mathrm{~km} / \mathrm{h}$ \\
\hline Doppler Shift & $29 \mathrm{~Hz}$ \\
\hline Location of non-zero Channel taps & {$[014142145]$} \\
\hline
\end{tabular}


when higher data constellation is used in simulation. Therefore, BPSK signal constellation is used $[32,33]$. Furthermore, the BER and MSE are used as figures of merit with various probability values of GMM channel i.e. $\emptyset=0, \emptyset=0.005$, and $\emptyset=0.05$.

\section{Results and Analysis}

In this paper, four- channel estimation schemes are tested in AWGN and GMM environments. In [8], different time domain channel estimation methods are performed based on the cyclic correlation method that required less complexity of computation and AWGN channel model was used. The AWGN channel model is used in various applications however the performance of channel estimation schemes deteriorates abruptly when it comes across impulsive noise. In the presented work four different channel estimation schemes are tested in GMM environment. Which can perform very well in both AWGN and GMM environment. An optimized method (Gauss-Seidel) is presented that has the less complexity with improved efficiency. In the proposed work four channel estimation schemes are tested (MMSE, DFT, GS and SOR) in terms of BER and MSE and shown that SOR method is more accurate than GS method.

Figure 3 shows the number of iterations for the SOR based TD CE method has been tested at $20 \mathrm{~dB}$ SNR, where the MSE of the estimated CIR is designed in contrast to the number of iterations. The legend AWGN $\emptyset=0$ represents in the AWGN channel. However, the GMM $\emptyset=$ $0.005, \varnothing=0.01$ and $\varnothing=0.05$ represent the probability values of GMM channel. It is verified that the CE schemes perform expressively worse when the impulsive noise is added in AWGN in Figure 3. Moreover, it shows that the GMM scheme is converged in the second iteration for different values of $\emptyset=0, \emptyset=0.005, \varnothing=0.01$ and $\varnothing=0.05$.

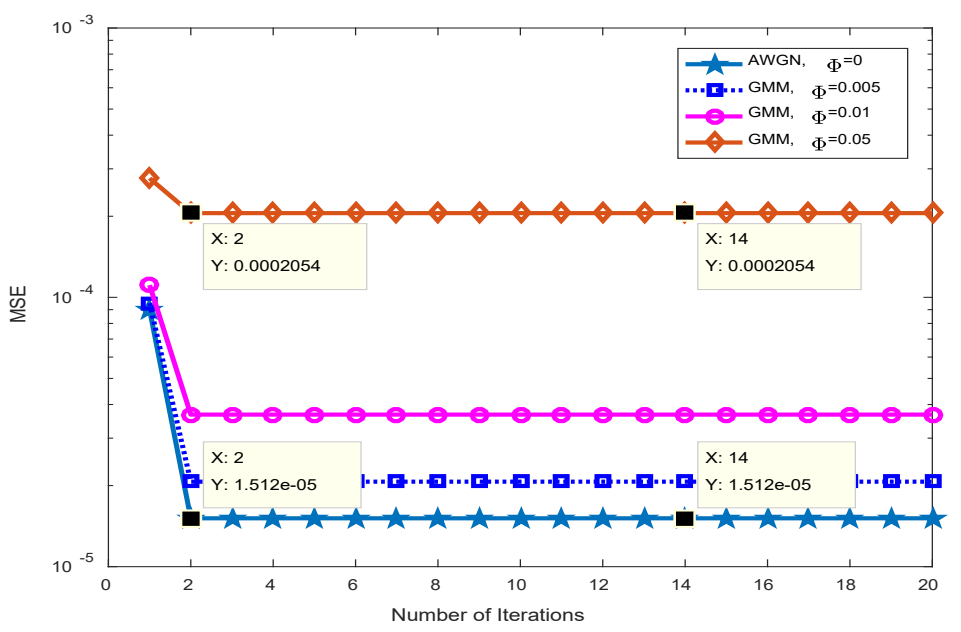

Figure 3. Number of Iterations at different values of $\emptyset$

In Figure 4, the MSE performance of conventional channel estimators (DFT, MMSE, GS) is compared with the SOR, $\omega=1.25$ in the AWGN channel where $\emptyset=0$. It is obvious that the SOR method performs much better than other estimators. It also shows that the difference of MSE between GS and SOR is dominant as compared to the high SNR regime. Because in a low SNR regime up to $5 \mathrm{~dB}$, the AWGN noise component is dominant as compared to correlation error. However, at higher SNR it is vice versa. Furthermore, the significant MSE difference can also be seen in Figure 4 at a high SNR regime between conventional and SOR. In Figures 5 and 6, the overall MSE of four estimators increases whenever these estimators expose in the GMM environment. However, the SOR shows the lowest MSE among all other estimators. It is also 
Saveeta Bai, et al.

investigated that the difference of MSE between GS and SOR is decreased because the GMM noise component is dominant as compared to AWGN and correlation error.

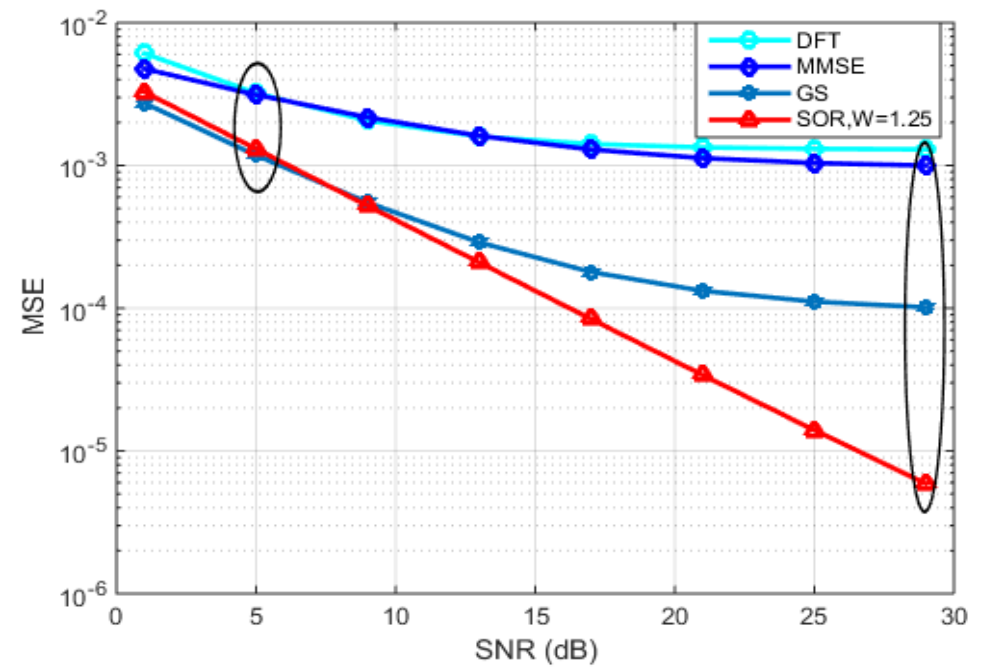

Figure 4. SNR's performance in AWGN channel using $\emptyset=\mathbf{0}, \mathbf{T}=\mathbf{0}$

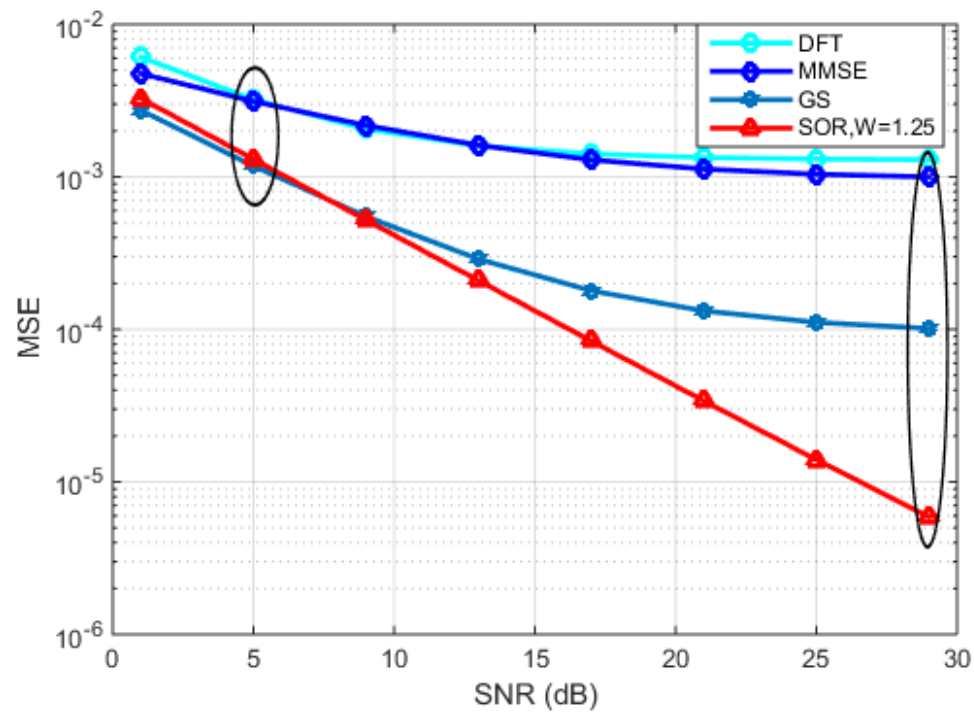

Figure 4. Performance of SNR in GMM channel using

$$
\emptyset=0.005, T=100
$$




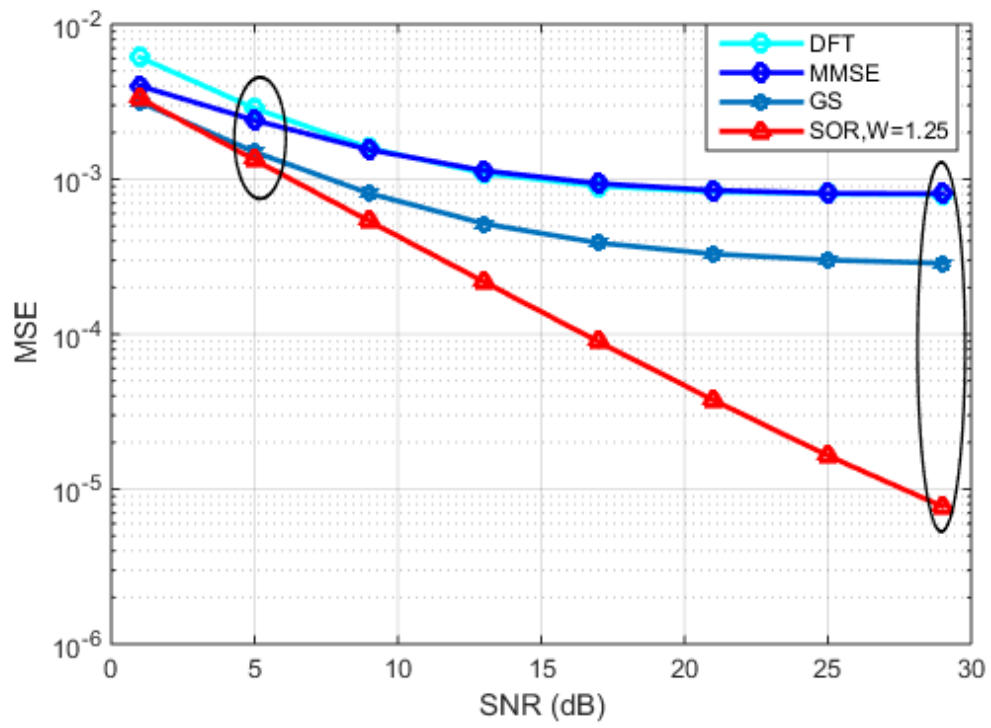

Figure 6. Performance of SNR in GMM channel using

$$
\emptyset=0.05, T=100
$$

Figures 7 and 8 show the BER curves under GMM environments in which SOR shows the lowest BER as compared to the other schemes. The convolution code that are utilized in BER performance, in such way that with the code rate of $1 / 2$ and the generator polynomials of mother code $\mathrm{G}_{1}=171_{\text {ОСт }}$ and $\mathrm{G}_{2}=131_{\text {ост }}$. It is also observed in Figures 8 and 9 that when $\varnothing=\mathbf{0 . 0 0 5}$ and $\emptyset=\mathbf{0 . 0 5}$ the BER of all the estimators is increased; however, the SOR sustains at the lowest value among all. $\mathrm{P}$

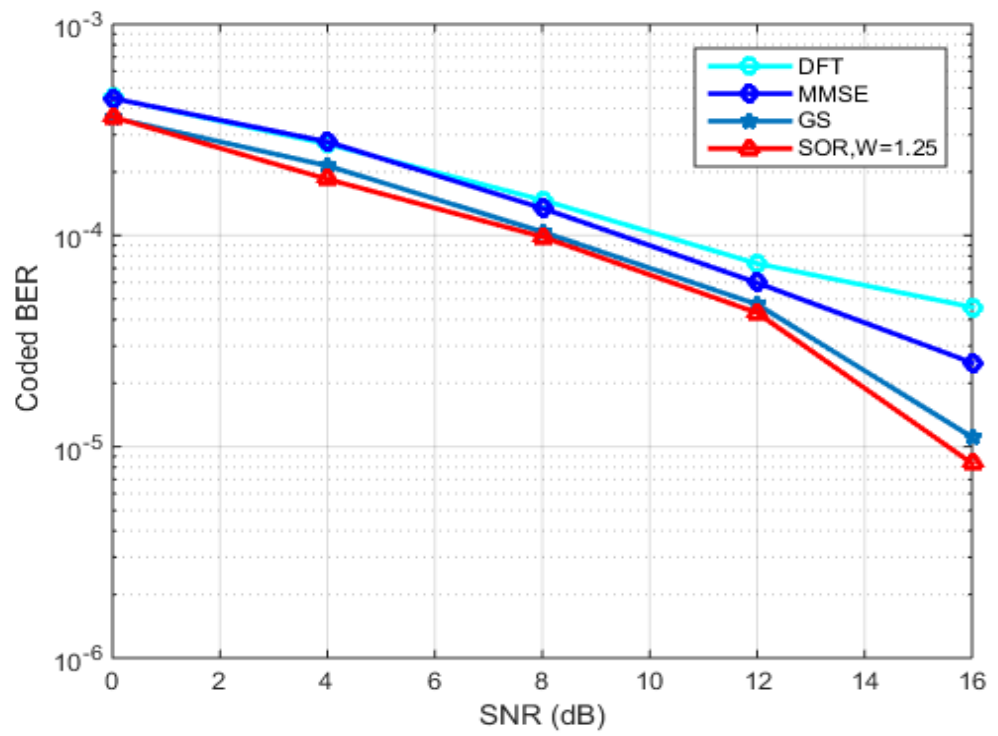

Figure 7. Performance of the CE in AWGN channel using $\emptyset=\mathbf{0}, \mathbf{T}=\mathbf{0}$ 


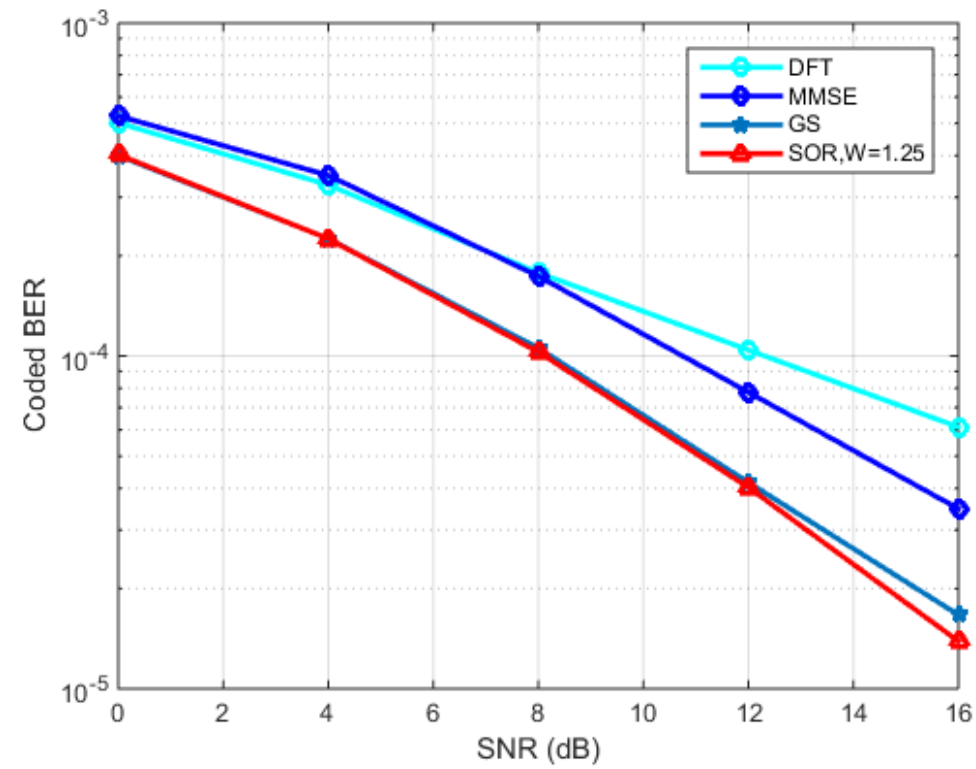

Figure 8. Performance of the CE in GMM channel using $\varnothing=\mathbf{0 . 0 0 5}, \mathbf{T}=\mathbf{1 0 0}$

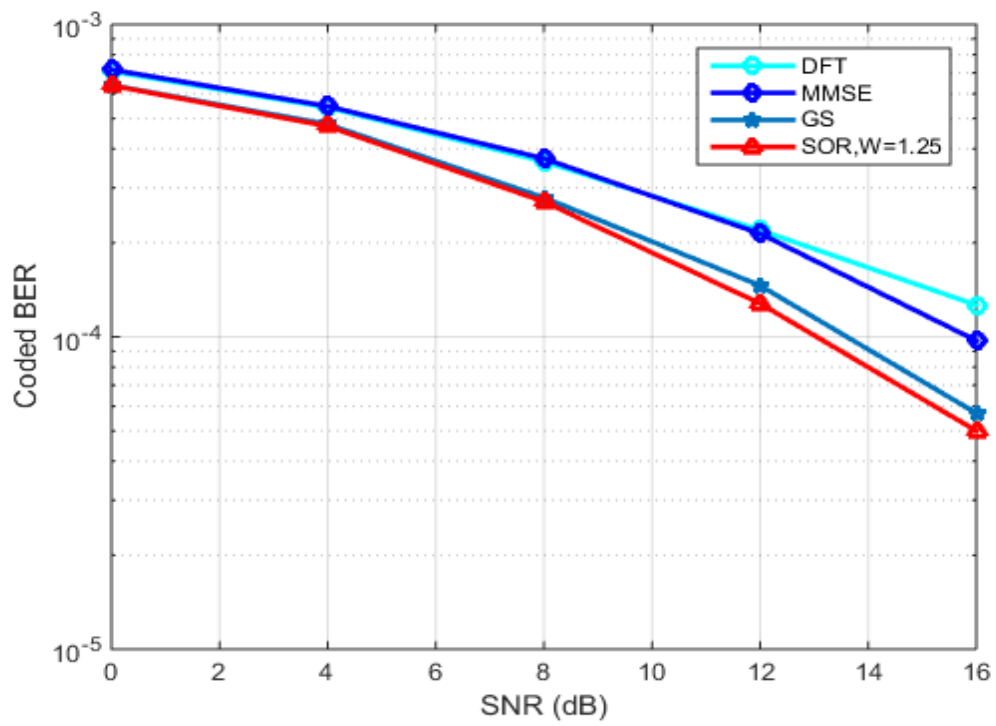

Figure 9. Performance of the CE in AWGN channel with $\emptyset=\mathbf{0 . 0 5}, \mathbf{T}=\mathbf{0}$

\section{Conclusion}

In this work, four diverse wireless channel estimation schemes are tested for AWGN and GMM environments. Furthermore, the performance of channel estimators is calculated with regard to MSE and BER. The analysis shows that the performance of all channel estimation schemes is deteriorated due to the presence of an impulsive component in the GMM environment. However, the SOR scheme has much better performance as compared to conventional DFT, MMSE and GS schemes. The SOR scheme has achieved improved performance by utilizing the high convergence rate due to the selection of an appropriate relaxation factor. The possible extension of this study is to analyze the hardware realization of 
GS and SOR schemes on FPGA implementation. FPGA implementation can provide reconfigurability, resources allocation, symmetric, and the timing diagram. Moreover, in the presented work channel estimation schemes are tested in AWGN and GMM environment it can be tested in the colored noise as a part of extension to this study in future.

\section{References}

[1] V. Kansal and S. Singh, "Capacity analysis of maximal ratio combining over Beaulieu-Xie fading," Annals of Telecommunications, vol. 76, pp. 43-50, 2021.

[2] O. E. Ijiga, O. O. Ogundile, A. D. Familua, and D. J. Versfeld, "Review of channel estimation for candidate waveforms of next generation networks," Electronics, vol. 8, p. 956, 2019.

[3] F. J. Martín-Vega and G. Gómez, "A Low-Complexity Pilot-Based Frequency-Domain Channel Estimation for ICI Mitigation in OFDM Systems," Electronics, vol. 10, p. 1404, 2021.

[4] H. A. Abbood, H. F. Khazal, and T. M. Jamel, "Time Domain Pilot-Based Channel Estimation (TDPCE) Using Kalman Filtering for OFDM System," in IOP Conference Series: Materials Science and Engineering, 2021, p. 012064.

[5] A. Saci, A. Al-Dweik, and A. Shami, "On the Spectral Efficiency of Blind Channel Estimation and Synchronization Techniques," arXiv preprint arXiv:1702.02366, 2017.

[6] N. Suga and T. Furukawa, "Scalar Ambiguity Estimation Based on Maximum Likelihood Criteria for Totally Blind Channel Estimation in Block Transmission Systems," IEEE Transactions on Wireless Communications, vol. 20, pp. 2608-2620, 2020.

[7] H. Qu, G. Liu, Y. Wang, Q. Chen, C. Yi, and J. Peng, "A time-domain approach to channel estimation and equalization for the SC-FDM system," IEEE Transactions on Broadcasting, vol. 65, pp. 713-726, 2019.

[8] A. M. Khan, V. Jeoti, M. U. Rehman, M. T. Jilani, and M. Zakariya, "A cyclic correlationbased time domain channel estimation scheme," in 2016 6th International Conference on Intelligent and Advanced Systems (ICIAS), 2016, pp. 1-5.

[9] I. Kalambi, "A comparison of three iterative methods for the solution of linear equations," Journal of Applied Sciences and Environmental Management, vol. 12, 2008.

[10] J. Li, S. I. Saeed, T. Yang, Y. Xie, and G. Zhang, "Low-Complexity MMSE Precoding Based on SSOR Iteration for Large-Scale Massive MIMO Systems," in Advances in Intelligent Information Hiding and Multimedia Signal Processing, ed: Springer, 2021, pp. 381-388.

[11] S. Karunanithi, N. Gajalakshmi, M. Malarvishi, and M. Saileshwari, "A Study on comparison of Jacobi, Gauss-Seidel and SOR methods for the solution in system of linear equations," Int. J. of Math. Trends and Technology,(IJMTT), vol. 56, 2018.

[12] Z. Albataineh, K. Hayajneh, H. B. Salameh, C. Dang, and A. Dagmseh, "Robust massive MIMO channel estimation for 5G networks using compressive sensing technique," AEUInternational Journal of Electronics and Communications, vol. 120, p. 153197, 2020.

[13] A. M. Khan, V. Jeoti, M. Rehman, and M. T. Jilani, "Noise power estimation for broadcasting OFDM systems," in 2017 IEEE 30th Canadian Conference on Electrical and Computer Engineering (CCECE), 2017, pp. 1-6.

[14] M. S. Miah, M. Rahman, T. K. Godder, B. Singh, and M. T. Parvin, "Performance Comparison of AWGN, Flat Fading and Frequency Selective Fading Channel for Wireless Communication System using 4 QPSK," 2011.

[15] C.-K. Wen, S. Jin, K.-K. Wong, J.-C. Chen, and P. Ting, "Channel Estimation for Massive MIMO Using Gaussian-Mixture Bayesian Learning," IEEE Transactions on Wireless Communications, vol. 14, pp. 1356-1368, 2015.

[16] G. Gui, L. Xu, and N. Shimoi, "Stable sparse channel estimation algorithm under nonGaussian noise environments," in 2015 21st Asia-Pacific Conference on Communications (APCC), 2015, pp. 561-565. 
[17] M. Muthukrishnan, P. G. Sudheesh, and M. Jayakumar, "Channel estimation for high mobility MIMO system using particle filter," 2016 International Conference on Recent Trends in Information Technology (ICRTIT), pp. 1-6, 2016.

[18] C. Soltanpur, K. M. Rabie, B. Adebisi, and A. Wells, "Masreliez-Equalized VOFDM in Non-Gaussian Channels: Power Line Communication Systems," IEEE Systems Journal, vol. 12, pp. 2803-2811, 2018.

[19] D. Shrestha, X. Mestre, and M. Payaró, "Asynchronous impulsive noise mitigation based on subspace support estimation for PLC systems," in 2016 International Symposium on Power Line Communications and its Applications (ISPLC), 2016, pp. 1-6.

[20] S. Laksir, A. Chaoub, and A. Tamtaoui, "Iterative algorithms for impulsive noise reduction in OFDM-based power line communications," International Journal of Embedded Systems, vol. 11, pp. 147-162, 2019.

[21] A. Lukezic, T. Vojir, L. “Cehovin Zajc, J. Matas, and M. Kristan, "Discriminative correlation filter with channel and spatial reliability," in Proceedings of the IEEE Conference on Computer Vision and Pattern Recognition, 2017, pp. 6309-6318.

[22] D. Pena, C. Lima, M. Dória, L. Pena, A. Martins, and V. Sousa, "Acoustic impulsive noise based on non-gaussian models: an experimental evaluation," Sensors, vol. 19, p. 2827 , 2019.

[23] S. Dahiya and A. K. Singh, "Channel estimation and channel tracking for correlated blockfading channels in massive MIMO systems," Digital Communications and Networks, vol. 4, pp. 138-147, 2018.

[24] S. Wesemann, "Method for channel estimation in a wireless communication system, communication unit, terminal and communication system," ed: Google Patents, 2018.

[25] A. Khan, V. Jeoti, and M. Zakariya, "Improved pilot-based LS and MMSE channel estimation using DFT for DVB-T OFDM systems," in 2013 IEEE Symposium on Wireless Technology \& Applications (ISWTA), 2013, pp. 120-124.

[26] H. Hamlili, S. Kameche, and A. Abdelmalek, "SaS noise suppression for OFDM wireless communication in rayleight channel," International Journal of Electrical and Computer Engineering, vol. 10, p. 2003, 2020.

[27] T. S. Rappaport, Wireless communications: principles and practice vol. 2: prentice hall PTR New Jersey, 1996.

[28] M. Sandell and O. Edfors, A comparative study of pilot-based channel estimators for wireless OFDM: Luleå tekniska universitet, 1996.

[29] J.-J. Van De Beek, O. Edfors, M. Sandell, S. K. Wilson, and P. O. Borjesson, "On channel estimation in OFDM systems," in 1995 IEEE 45th Vehicular Technology Conference. Countdown to the Wireless Twenty-First Century, 1995, pp. 815-819.

[30] F. Rouissi, A. J. H. Vinck, H. Gassara, and A. Ghazel, "Improved impulse noise modeling for indoor narrow-band power line communication," AEU-International Journal of Electronics and Communications, vol. 103, pp. 74-81, 2019.

[31] L. Kewen, "Research of MMSE and LS channel estimation in OFDM systems," in The 2nd international conference on information science and engineering, 2010, pp. 2308-2311.

[32] S. S. Dash, F. Pythoud, B. Baeuerle, A. Josten, P. Leuchtmann, D. Hillerkuss, et al., "Receiver Algorithm for Decoding Constellation Modulation," in Signal Processing in Photonic Communications, 2016, p. SpTu1F. 3.

[33] S. S. Dash, F. Pythoud, D. Hillerkuss, B. Baeuerle, A. Josten, P. Leuchtmann, et al., "Constellation modulation-an approach to increase spectral efficiency," Optics express, vol. 25, pp. 16310-16331, 2017. 


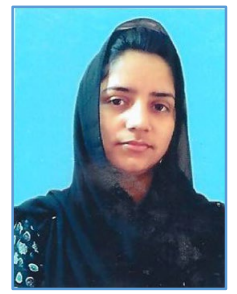

Saveeta Bai received her B.S. degree in Electronic Engineering from Sir Syed University of Engineering and Technology, Karachi, Pakistan in 2016 and the M.S degree in Electronics Engineering from Sir Syed University of Engineering and Technology, Karachi, Pakistan in 2019. Moreover, she has served as Research Assistant in Department of Electronic Engineering, Sir Syed University of Engineering and Technology, Karachi, Pakistan since 2017 to 2019. Currently conducting research on Deep Learning for Millimeter wave.

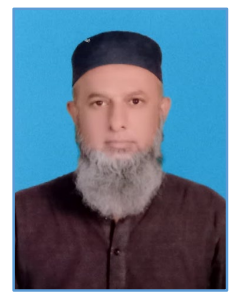

Abid Muhammad Khan received his B.S. degree in electronic engineering from NED University of Engineering and Technology, Karachi, Pakistan. He accomplished his Ph.D. in electronics engineering from department of electrical and electronics engineering, Universiti Teknologi PETRONAS, Malaysia. He is a life time member of Pakistan Engineering Council and professional member of IEEE for last 12 years. Currently he is the associate professor in Electrical Engineering Department, Sir Syed University of Engineering and Technology, Karachi.

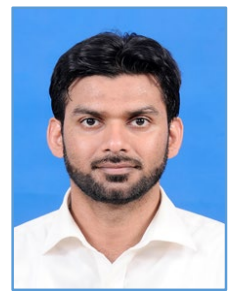

Muhammad Rauf received his B.S. degree in electronic engineering from Dawood University of Engineering and Technology, Karachi, Pakistan in 2008 and the M.S degree in electronics engineering from Universiti Malaysia Pahang, Malaysia in 2012. He accomplished his Ph.D. in electrical and electronics engineering from Universiti Teknologi PETRONAS, Malaysia in 2017. He is a life time member of Pakistan Engineering Council and member of IEEE. Currently he is serving as Assistant Professor in Department of Electronic Engineering, Dawood University of Engineering and Technology,

Karachi, Pakistan

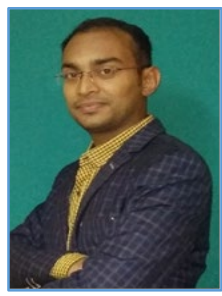

Suresh Kumar is doing his $\mathrm{PhD}$ in Electronic Engineering from The Polytechnic University of Catalonia, Barcelona Spain. Moreover, he has served as lecturer in the Science \& Technology Department of Indus University Karachi Pakistan since 2016 to 2020. He completed his BS in electronic engineering in 2014 from Sir Syed University of Engineering \& Technology Pakistan, furthermore he completed his Master of Engineering in Electrical Engineering in 2019 from Hamdard University Pakistan.

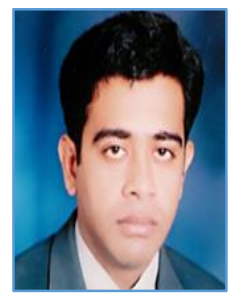

Haresh Kumar received his B.S. degree in Electronic Engineering from Sir Syed University of Engineering and Technology, Karachi, Pakistan in 2010 and the M.S degree in Electronics Engineering from Sir Syed University of Engineering and Technology, Karachi, Pakistan. Currently he is serving as Research Assistant in Department of Electronic Engineering, Sir Syed University of Engineering and Technology, Karachi, Pakistan. 